\title{
Pendientes de los servicios farmacéuticos comunitarios en México que limitan el uso racional de los medicamentos
}

\author{
María T. Espinosa Roque ${ }^{1}$, Mariana Ornelas Perea ${ }^{2}$ \\ 1. Profesora de Farmacología. Coordinación de Ciencias Básicas. Escuela de Medicina de la Universidad Cuauhtémoc Plantel Aguascalientes. México. \\ 2. Alumna de la Licenciatura Médico Cirujano Integral. Escuela de Medicina. Universidad Cuauhtémoc Plantel Aguascalientes. México.
}

\section{PALABRAS CLAVE}

Servicios farmacéuticos, uso racional de medicamentos, farmacia comunitaria, profesional farmacéutico

\section{ABREVIATURAS}

ANAFARMEX: Asociación Nacional de Farmacias

Mexicanas

COFEPRIS: Comisión

Federal para la Protección de Riesgos Sanitarios

FIP: Federación

Farmacéutica Internacional

OMS: Organización

Mundial de la Salud

OPS: Organización

Panamericana de la Salud

PFN: Política Farmacéutica

Nacional

PET: grupo de trabajo de Educación Farmacéutica

Global (acrónimo en inglés)

PRM: problemas

relacionados con

medicamentos

RAM: reacciones adversas

a los medicamentos

SF/APS: servicios

farmacéuticos basados en

la atención primaria de

salud.

SF: servicio farmacéutico

URM: uso racional de

medicamentos

\section{KEYWORDS}

Pharmaceutical services, rational use of drugs, community pharmacy, pharmacist professiona

\section{RESUMEN}

La posición de la Organización Mundial de la Salud (OMS) y Organización Panamericana de la Salud (OPS) es que los países de la región de las Américas implanten un modelo de servicios farmacéuticos que garantice una atención integral en la salud de la población respecto a los medicamentos y define el uso racional de medicamentos cuando "los pacientes reciben la medicación adecuada a sus necesidades clínicas, en las dosis correspondientes a sus requisitos individuales, durante un período de tiempo adecuado y al menor coste posible para ellos y para la comunidad".

En México el uso inadecuado de los medicamentos representa un problema grave de salud que conlleva a una accesibilidad no equitativa, incrementa los costos de tratamientos y obliga al paciente a convivir con problemas relacionados con la medicación; las instituciones de salud han realizado esfuerzos por promover una política farmacéutica nacional en congruencia con las recomendaciones de la OMS, sin embargo no se ha tenido el impacto en los indicadores de salud. Por otra parte los profesionales farmacéuticos por diversos factores no influyen como líderes en el desarrollo de un servicio integral orientado al beneficio del paciente (instrucciones para su utilización y orientación ante la detección de problemas relacionados con su manejo) y a su vez el modelo de farmacia comunitaria orienta el servicio más hacia un fin comercial que sanitario. La ciudadanía se mueve entre la expectativa y la incertidumbre derivada de la información recibida de los farmacéuticos, los médicos, los medios de comunicación y de los propios laboratorios farmacéuticos.

Darrings community pharmaceutical services in Mexico that limit the rational use of medicines

\begin{abstract}
The position of the World Health Organization and Pan American Health Organization is that the countries of the Americas region to implement a model of Pharmaceutical Services to ensure comprehensive care in the health of the population regarding drug and defines Use rational Drug when "patients receive appropriate medication to their clinical needs, in appropriate doses to individual requirements, for an adequate period of time and at the lowest possible cost for them and for the community."

In Mexico misuse of drugs is a serious health problem that leads to an inequitable accessibility, increased treatment costs and requires the patient to live with drug-related problems; Health institutions have made efforts to promote a National Drug Policy in line with WHO recommendations, however has not had the impact on health indicators. Furthermore pharmaceutical professionals by various factors do not influence as leaders in the development of a comprehensive service-oriented patient benefit (instructions for use and guidance upon detection of problems related to their management) and in turn the model Community Pharmacy orients the health service towards that commercial purpose. Citizenship moves between expectation and uncertainty arising from the information received from pharmacists, doctors, the media and pharmaceutical laboratories.
\end{abstract}

Financiación: Ninguna.

Conflicto de intereses: Las autoras declaran no existir conflicto de intereses en relación con el contenido del artículo. Contribución a la autoría: MTER diseñó el estudio, realizó la investigación, participó en la redacción del manuscrito y revisó el contenido final. MOP contribuyó a la redacción del manuscrito y revisó el contenido final.

Cite este artículo como: Espinosa MT, Ornelas M. Pendientes de los servicios farmacéuticos comunitarios en México que limitan el uso racional de los medicamentos. Farmacéuticos Comunitarios. 2016 Sep 30; 8(3):29-34. doi:10.5672/FC.2173-9218.(2016/Vol8).003.06

Autora para correspondencia: Maria T. Espinosa Roque (tere.espinosaroque@gmail.com).

ISSN 1885-8619 @SEFAC (Sociedad Española de Farmacia Familiar y Comunitaria). Todos los derechos reservados. 


\section{Introducción}

Los medicamentos son una herramienta fundamental en la terapéutica moderna, que al utilizarse después de un método diagnóstico adecuado permiten la prevención, curación, atenuación y tratamiento de las enfermedades. Sin embargo, cuando estos se utilizan de manera inapropiada se convierten en una amenaza para la salud (1). En todo el mundo, más del $50 \%$ de todos los medicamentos se recetan y se venden de forma inadecuada, siendo algunos tipos frecuentes de uso irracional, la polifarmacia y automedicación inadecuada, a menudo con medicinas que requieren receta médica, suponiendo tanto un desperdicio de recursos y un considerable perjuicio al paciente en cuanto a la falta de resultados positivos (2).

En México los estudios que puedan relacionar las evidencias que existen respecto al uso irracional de medicamentos con efectos sobre indicadores de salud son limitados. Algunos trabajos epidemiológicos realizados indican que la automedicación representa un problema grave de salud pública, obteniendo como resultado resistencia a antibióticos, ineficacia terapéutica, Reacciones Adversas a los Medicamentos (RAM), presencia de efectos tóxicos e interacciones farmacológicas severas, reduciendo la calidad de vida de los pacientes $(3,4)$. En este sentido, la farmacia comunitaria es el espacio de oportunidad para la intervención en el ámbito de prevención y promoción de la salud donde el profesional farmacéutico es el responsable de orientar este servicio de salud, más hacia el bienestar del paciente que al medicamento $(3,5,6)$, para lo cual debe desarrollar competencias en el campo de la investigación, la gestión del conocimiento, el cumplimiento de la legislación, la promoción de la educación permanente de recursos humanos y su propia capacitación continua (6).

\section{Antecedentes}

La profesión farmacéutica en México a través de la historia ha encarado una difícil situación. Por citar algunas fechas, en 1921 se autorizó la existencia de farmacia sin responsable titulado, así como la posición y responsiva de varias farmacias para un responsable. No fue sino hasta 1942 que el Reglamento para Droguerías, Farmacias y demás establecimientos similares estableció las horas mínimas de estancia en la farmacia del responsable autorizado, se aceptaron prácticos farmacéuticos como responsables y se definieron requisitos para ocupar dichos puestos. En 1973 por primera vez se prohibió sin excepciones el ejercicio de las profesiones sanitarias sin el título reconocido por la Secretaría de Educación Pública y el registro sanitario ante la Secretaría de Salubridad y Asistencia, sellando los requisitos legales (7). En el año 2009 se difundió como propuesta no regulatoria un Modelo Nacional de Farmacia planteando una adecuación profunda en la organización de la farmacia, del personal que las componen y de los procesos, precisando los aspectos básicos que deberán ser considerados por los profesionales para desarrollar los Procedimientos Normalizados de Operación, promoviendo el desarrollo de las farmacias y el Uso Racional de Medicamentos (URM), con un enfoque hacia el surtido de acuerdo a necesidades y fomento del conocimiento, así como la responsabilidad social del trabajo de los dispensadores (8).

La Comisión Federal para la Protección contra Riesgos Sanitarios (COFEPRIS) destaca el creciente fenómeno de las farmacias con consultorio, indicando que el $43 \%$ de las farmacias ofrece consultas médicas. Entre 2010 y 2012 las farmacias con consultorio crecieron el 130\% atendiendo mensualmente al 7\% de la población mexicana, pudiendo generar beneficios importantes para la aplicación estricta de los lineamientos de control de antibióticos de 2010, así como para la prevención de la automedicación. Sin embargo, es una preocupación que estos consultorios en ocasiones no están regulados, situación que induce la prescripción y adquisición de medicamentos innecesarios (9).

En la actualidad, diversos estudios sobre acceso y uso de medicamentos han evidenciado que los clientes de farmacias adquieren medicamentos sin prescripción médica; no cumplen con tratamientos de antibióticos o los usan inadecuadamente, no tienen acceso equitativo a los medicamentos destinados a enfermedades crónicas y reciben consejos inapropiados y dañinos sobre su farmacoterapia para patologías frecuentes por parte del farmacéutico. Lo anterior evidencia lagunas para dirigir prioridad de acciones sobre el uso irracional, acceso inadecuado a medicamentos y proporciona orientación importante acerca de principales vacíos en base a los conocimientos en México, áreas donde se requiere promover una mayor investigación y dirección de objetivos de trabajo (10).

\section{Los servicios \\ farmacéuticos basados en la atención primaria de la salud}

Como una estrategia para el fortalecimiento de los sistemas de salud, la Organización Mundial de la Salud (OMS) y la Organización Panamericana de la Salud (OPS) han propuesto la implantación de un modelo de los servicios farmacéuticos basados en la Atención Primaria de la Salud (SF/ APS) $(3,6)$, han recomendado a los países desarrollar una Política Farmacéutica Nacional (PFN) que incorpore metas y acciones establecidas por el gobierno y coordinadas entre los actores del campo farmacéutico para asegurar la calidad de los medicamentos, lograr el acceso a los mismos y fomentar su uso racional. Destacan que los elementos esenciales para que los SF/APS garanticen lo anterior son: el acceso a los medicamentos, la seguridad y eficacia, el seguimiento y evaluación de utilización, la obtención y difusión de la información de medicamentos y la educación continua a los miembros del equipo de salud, el paciente y la comunidad (11).

El Servicio Farmacéutico (SF) se define como el "conjunto de acciones en el sistema de salud que buscan garantizar la atención integral, integrada y continua de las necesidades y problemas de la salud de la población tanto individual como colectiva, teniendo el medicamento como uno de los elementos esenciales, contribuyendo a su acceso equitativo y uso racional" $(6,11,12)$. Las competencias específicas para este servicio se organizan en funciones tales como: las vinculadas al paciente, la familia y la comunidad como estrategia central; las vinculadas a la investigación y gestión del conocimiento; y aquellas sujetas al desempeño profesional $(10,13)$. Incluso pueden contribuir en la solución de problemas de salud a 
través de acciones, promoviendo la adherencia a tratamientos, llevando a cabo intervenciones cuando se identifiquen Problemas Relacionados con los Medicamentos (PRM) y coadyuvar a la reducción de errores de medicación, entre otras (11).

En México este servicio se caracteriza por tener una laxa regulación sanitaria que limita el cumplimiento a cabalidad de los ordenamientos legales en lo que respecta a la venta o suministro de medicamentos, ya que en las farmacias los medicamentos incluidos en el Artículo 226, de la Ley General de Salud, fracciones III y IV4, frecuentemente se expenden sin la presentación de una receta médica, excepto en el caso de sustancias psicotrópicas o estupefacientes, lo que implica violación a la legislación sanitaria $(3,12)$. Hay una carencia de personal profesional en las farmacias para orientar el uso de los medicamentos (4) y su perfil de formación actual es muy variado, unido a que atienden más de una farmacia; la farmacia mexicana no considera necesaria la vigilancia ni pericia del profesional en la dispensación (7) y hasta ahora los distintos elementos relacionados con una PFN están dispersos en un marco legal fragmentado y en los Programas Sectoriales difundidos cada sexenio (3). El documento de la Secretaría de Salud Hacia una politica farmacéutica integral para México se sigue revisando y refinando al tiempo que se implementa, lo que limita los esfuerzos del país en cuanto a la accesibilidad de medicamentos por la población y más aún, la evaluación de sus resultados (14).

\section{El papel del \\ farmacéutico en el contexto de la farmacia comunitaria}

El abordaje del desempeño del SF tiene una repercusión directa en el quehacer de los profesionales que entregan el mismo. De un papel con un marcado énfasis en tareas administrativas de logística de medicamentos $\mathrm{y}$ en la atención curativa, se requiere que las funciones que se han venido cumpliendo y las nuevas que se proponen tengan como enfoque la salud y los estilos de vida $(5,11)$.

La tendencia mundial del ejercicio profesional del farmacéutico es centrar su objetivo en el cuidado del paciente, formando parte activa del equipo de salud y proporcionando los SF necesarios para asegurar que éste pueda acceder a terapias oportunas, seguras y eficaces $(11,12,15)$, identificar, corregir y prevenir los PRM para promover su uso racional, mejorando así el estado general de la salud de la población $(11,16)$.

Se conoce en la historia sobre las propuestas de los farmacéuticos en el país para impugnar el atraso de la legislación mexicana, en aras de defender el espacio laboral de su quehacer en beneficio de la sociedad sin que se atendieran sus alertas, lo que provocó que se fueran a trabajar a otros puestos oficiales, abandonando la botica donde su trabajo no era ni reconocido ni bien remunerado. Su presencia incluso en las farmacias quedó reducida a unas cuantas horas para cumplir su papel de responsables. Esto detonó la transformación profesional de farmacéutico a químico-farmacéutico (7).

En las farmacias comunitarias de México laboran profesionales que atienden varias farmacias cada uno y que han sido designados por los titulares de licencias sanitarias como responsables sanitarios, debiendo contar con título de farmacéutico industrial, químico industrial, homeópata o médico (17). No obstante a esta amplia gama de perfiles, la Asociación Nacional de Farmacias Mexicanas (ANAFARMEX) ha reportado la carencia de profesionales farmacéuticos, mencionando que existe un 0,57\% dentro de las cifras de más de 120.000 operadores que laboran en las farmacias del país (18).

Los farmacéuticos deben orientarse a la polifarmacia, el uso inadecuado de antimicrobianos, uso excesivo de inyecciones en casos en los que serían factibles las formulaciones orales y el recetado no acorde con las directrices clínicas y la automedicación inadecuada (2). Asimismo, cada vez se tiene más conciencia de que facilitar únicamente el medicamento a los usuarios no es suficiente para alcanzar los objetivos del tratamiento, por lo que estos profesionales precisan condiciones que los lleven a un cambio en el ejercicio de sus funciones, ser reconocidos como profesionales sanitarios cuya primera preocupación sea el bienestar del paciente, con accesibilidad a que puedan consultar sus problemas relacionados con la sa- lud para ser resueltos a nivel de atención primaria (18). Son profesionales sanitarios que disponen de autorización para que gestionen la dispensación de medicamentos a los usuarios y realicen tareas adecuadas para garantizar su seguridad y eficacia (19). Igualmente, tendrán como funciones las relacionadas con la Atención Farmacéutica al proporcionarle opciones al paciente para sustituir un medicamento hacia el genérico, revisar y validar prescripciones de acuerdo a aspectos terapéuticos, sociales, económicos y legales, así como asesorar al paciente para que obtenga el máximo beneficio de la terapia; promover un uso racional y económico, prevenir contraindicaciones y RAM y dar seguimiento farmacoterapéutico para comprobar los efectos de la medicación, indagar sobre problemas, consecuencias y pertinencia del tratamiento en el período de vigencia de la prescripción médica así como otros resultados de la farmacoterapia $(11,20)$.

Las farmacias comunitarias son, ante todo, establecimientos de salud que, si logran brindar servicios adecuados, constituyen la fuente del primer contacto y una importante puerta de entrada al sistema de salud. Con una frecuencia significativa estos servicios son incluso el único contacto del usuario con dicho sistema, el más cercano, con disponibilidad de horario para la atención tanto de pacientes como de personas sanas que solicitan medicamentos necesarios, también aquellos que los ponen en riesgo, que son innecesarios o abusivos y van en busca de consejos sobre salud (5).

Las intervenciones por el farmacéutico pueden ser prometedoras para mejorar resultados importantes de un buen uso de medicamentos (15). Las revisiones sobre su eficacia han demostrado que mejoran la adherencia, el uso por los pacientes, el conocimiento sobre la base de la información y reducen los eventos adversos y mortalidad (21). Un estudio ha demostrado, respecto a la confianza en los medicamentos genéricos, que la información verbal y escrita sobre sus ventajas e inconvenientes hace que los pacientes acepten el cambio en el 98,9\% de los casos (22). Otro ejemplo reportado por la COFEPRIS es que posterior a que en 2010 se diera la indicación de que los medicamentos antibióticos solo deberán venderse 
con receta médica, las cifras de la venta de antibióticos bajaron un 36\% y mediante la revisión de las recetas retenidas por las farmacias se pudo conocer que el $46 \%$ de 79.177 recetas presentaba prescripción incompleta al faltar datos importantes como dosis, duración del tratamiento, presentación y vía de administración (13).

Un buen servicio de gestión de la seguridad del medicamento y de farmacovigilancia es un requisito imprescindible para la detección precoz de los riesgos asociados con medicamentos, la prevención de reacciones adversas a estos y para conseguir la mejor relación beneficio/riesgo con una terapia segura y efectiva (20).

A pesar de todas las recomendaciones dadas por la OMS y la Federación Farmacéutica Internacional (FIP) acerca del papel del farmacéutico en los Servicios de Salud, México a través de la historia ha sido un país retrasado en el campo del ejercicio profesional farmacéutico y el papel de la farmacia comunitaria respecto a otros países (7).

\section{Las competencias de la profesión farmacéutica}

La Federación Mundial de Educación Médica plantea que las normas internacionales de competencias pueden ser definidas para la educación médica y farmacéutica básica teniendo en cuenta variaciones de los países como cultura, sistema de salud, condiciones socioeconómicas pero con bases científicas universales. Por estas razones la Federación Farmacéutica Internacional (FIP) y el grupo de trabajo de educación de farmacia global (PET) ha trabajado de acuerdo a la búsqueda de la literatura y encuesta mundial para el desarrollo de un marco legal de competencias de los SF que sirva de mapeo que prospera de acuerdo a como progresa la profesión y que es aplicable en todo el mundo (21).

En distintos países que presentan mapas de competencias, se realizó la revisión en los diferentes marcos en base a las competencias en salud pública, en el cuidado, organización y gestión de competencias y competencia profesional/personal, lo cual permitió que el PET junto a la Organización de Naciones Unidas para la Educación, la Ciencia y la Cultura (UNESCO) y la OMS formularan el plan de acción de Educación de Farmacia y la Educación Continua, basados en necesidades de educación y sostenibilidad de la fuerza de trabajo (23). Dichas competencias se clasifican en: competencia genérica, específica y humanística (5).

En el caso del papel del farmacéutico en el sistema de salud, la OMS ha detallado en su informe del grupo consultivo sobre la preparación del farmacéutico del futuro siete competencias mínimas y esenciales que debe demostrar en su desempeño en los sistemas de salud a nivel mundial y que fueron señaladas como las siete estrellas, refiriéndose a las responsabilidades de: proporcionador de salud como miembro del equipo de salud, tomador de decisiones y de asumir responsabilidad como comunicador, líder, gerente, aprendiz permanente y educador $(6,24,25,26)$.

En la actualidad, la Agenda de Salud para las Américas 2008-2017 identifica dentro de las áreas de acción: el acceso a medicamentos como un servicio de salud de la atención primaria de salud; el mejoramiento de los sistemas de información sanitaria a nivel nacional y local para facilitar la provisión de servicios de una forma integral y oportuna; acercar a las instituciones de formación y las de servicios de salud para una planificación de perfiles de los profesionales en perspectiva multidisciplinaria y fomentar y fortalecer la capacidad de investigación y utilización del conocimiento a nivel local (27).

La realidad en México es que el modelo de dispensación de medicamentos al que estamos migrando en el país es el de grandes cadenas que son atendidas por empleados con escasos conocimientos en la atención farmacéutica (7). Además, cabe destacar que no existe incentivo que estimule la educación continua puesto que no está considerada como un requisito para continuar desempeñando la profesión, por lo que se convierte en otro campo de acción para los objetivos de trabajo (13).

\section{Estrategias para un URM}

La OMS recomienda la aplicación de intervenciones que sean rectificadoras a nivel político, legislativo/ regulativo, cultural/educativo e investigativo. En el nivel político los países deberán apoyar las políticas multidisciplinarias de salud como política de Estado; en el legislativo se requiere transparencia en toda la cadena del medicamento, la venta de medicamentos en establecimientos autorizados para este fin y la limitación de incentivos financieros a los profesionales de salud por las compañías farmacéuticas; en el educativo el acceso a la información por parte de profesionales y la comunidad así como la implementación de programas educativos de pregrado y postgrado. En el nivel de investigación se deberán impulsar las mismas en las instituciones educativas y científicas (28).

En los informes del Plan Nacional para los sexenios de 2007 a 2012 y 2013 a 2018 para la protección de riesgos sanitarios, presentados por las autoridades gubernamentales en México, se definen los riesgos por acceso inadecuado que llevan al deterioro de la salud, entre ellos: vigilancia deficiente de las farmacias que llevan al mal uso del medicamentos y al comercio ilegal, la automedicación desinformada, la ignorancia del consumidor (2), malas prácticas de dispensación y venta incontrolada por internet (29). Además, la omisión de la exigencia de la receta médica ha dado lugar a una práctica cotidiana de autoprescripción, donde se carga al consumidor con la enorme responsabilidad de garantizar el uso seguro y eficiente de los medicamentos (3).

No obstante a estos diagnósticos las líneas de acción de los programas se centran en la accesibilidad, la vigilancia y el control sanitario (29).

En el país la automedicación es una práctica potenciada por la inaccesibilidad de un alto porcentaje de la población a asistencia sanitaria, las prácticas de mercadotecnia, publicidad y venta (especialmente la promoción que se realiza por medio de la televisión), la compra de medicamentos inapropiados y la adquisición de cantidades mayores que las necesarias (4).

Un reto adicional está conformado por los incentivos para la prescripción y dispensación de medicamentos que van en contra de mejorar el uso seguro y eficiente. En los últimos años el establecimiento de consultorios adjuntos a farmacias privadas ha generado un fuerte conflicto de interés por los médicos que las atienden, 
pues su salario proviene directa o indirectamente de ella (3).

Con el objetivo de mejorar la calidad y la rentabilidad de las terapias el gobierno ha puesto en marcha tres programas: farmacovigilancia, uso racional de medicamentos y farmacéuticos clínicos en hospitales, que deberán vincularse a otras estrategias y evaluar el impacto de su aplicación en el tiempo (11).

A pesar de los avances alcanzados en cuanto al desabasto y la calidad como la verificación sanitaria para evaluar el cumplimiento de Buenas Prácticas de Fabricación; la instrumentación de la Política de Genéricos Intercambiables; el aseguramiento de medicamentos que no llegaron a comercializarse y la modificación y actualización del marco legal y normativo (29), el uso irracional sigue siendo una realidad que conspira en contra de resultados positivos en los indicadores de salud $(3,9,29)$. Además, las instituciones académicas y sociedades civiles tienen en este problema un papel marginal y no es claro en qué instancia recae la obligación de una estrategia nacional de mejora sobre su uso eficiente y seguro (3).

Los pendientes limitantes en este sentido en los Servicios Farmacéuticos Comunitarios se resumen en:

- La carencia de profesionales con formación en áreas de servicios de la salud que laboren como responsables sanitarios $(3,4)$.

- El SF Comunitario tiene un enfoque curativo y no preventivo (9).

- La insuficiente capacitación del profesional farmacéutico $(3,12)$.

- Laxa regulación sanitaria $(3,4)$.

- Los elementos de una Política Farmacéutica Nacional están fragmentados en el marco legal y Programas Sectoriales de Salud $(3,12)$.

- Falta de aplicación de intervenciones rectificadoras a nivel político, legislativo/regulativo, cultural/ educativo e investigativo (28).

\section{Agradecimientos}

Al Dr. Javier Góngora Ortega por las críticas que orientaron la elaboración de la presente revisión.

\section{Referencias bibliográficas}

1. Ramos, G. Olivares, G. Guía para las buenas prácticas de prescripción: metodología para la prescripción ra- cional de medicamentos ministerio de salud de Chile, División de políticas públicas saludables y promoción, 1era edición, Santiago de Chile, 2010, pp. 1.

2. Quick, J, Hogerzeil, H. Promoción del uso racional de medicamentos: componentes centrales. Perspectivas políticas sobre medicamentos de la OMS. 2002;5(1): 1-6. Disponible en: http://apps.who.int/medicinedocs/ pdf/s4874s/s4874s.pdf

3. Wirtz, V, Dreser, A, Heredia, I. Retos y Oportunidades para el Desarrollo de la Política Farmacéutica Nacional en México. Salud Pública. 2013; 55 (3): 329-336. Disponible en: Wirtz y Dreser, 2013.

4. Gómez, 0, Leobardo, M, Et al. Estudio de automedicación en una farmacia comunitaria de la ciudad de Toluca. Revista Mexicana de ciencias farmacéuticas. 2009;40(1): 5-11. Disponible en: http://redalyc.uaemex. $\mathrm{mx} / \mathrm{src} /$ inicio/ArtPdfRed.jsp?iCve $=57940102$

5. Organización panamericana de la salud. Servicios farmacéuticos basados en la atención primaria de la salud. OPS/OMS. 2011;1(1): Disponible en: http://www.paho.org/hq/index. php?option=com_docmanttask=doc_viewEtgid=21582\&Itemid=

6. Grupo técnico para el desarrollo de competencias para los servicios farmacéuticos. Competencias del farmacéutico para desarrollar los servicios farmacéuticos basados en atención primaria de salud y buenas prácticas de farmacia. Conferencia panamericana de educación. 2013;1(1): 10-32.

7. Ortiz, M, Aceves, P. La Legislación farmacéutica en México: disposiciones sobre el ejercicio profesional, 19171973. Revista Mexicana de ciencias farmacéuticas. 2014;45(1). Disponible en: http://www.redalyc.org/articulo. oa? id=57932293004

8. Secretaría de Salud. COFEPRIS. Guía de manejo y asistencia en la dispensación de medicamentos e insumos para la salud en farmacias. 2014. p: 2. Disponible en: http://www.cofepris.gob.mx/Documents/LoMasReciente/16042014.pdf

9. Peña, E. Programa sectorial de salud 2013-2018. Diario oficial de la federación. 2013;1(1): 12. Disponible en: http://portal.salud.gob.mx/contenidos/conoce_salud/prosesa/pdf/programaDOF.pdf

10. Wirtz, V, Reich, M, Flores, R, Dreser, A. Systematic review of research on access and use. Salud pública de México. 2008; 50(1):472-474.

11. OMS, OPS. Servicios farmacéuticos basados en la atención primaria de salud. Documento de posición de OMS/OPS. 2012;1(1): 29-49. Disponible en: http://www.paho.org/hq/ index.php?option $=$ com_docmancttask=doc_view\&tgid $=21582$ CIItemid $=$

12. Toscano, M. Comisión Federal para la Protección contra Riesgos Sanitarios. Secretaria de Salud. 20072012;1(1): 38, 39, 41, 43, 44, 45, 47. Disponible en: http://www.cofepris. gob.mx/cofepris/Documents/QueEsCOFEPRIS/prgaccion.pdf

13. ISDB. Prescripción, Farmacia y Utilización. Investigaciones comisión federal para la protección de riesgos sanitarios. 2012;1(1). Disponible en: http:// www.saludyfarmacos.org/lang/es/ boletin-farmacos/boletines/nov2012/ comisin-federal-para-la-proteccin-de-riesgos-sanitarios/

14. Salud pública de México/vol. 50, suplemento 4. Cuernavaca 2008 Políticas farmacéuticas en México: que 20 años no es nada* (pág. 423-425). Portal de información-medicamentos esenciales y productos de salud. Un recurso de la OMS. Disponible en: http://apps.who.int/medicinedocs/documents/s19899es/s 19899es.pdf

15. OMS, OPS. Propuesta de plan básico de educación farmacéutica y competencias del farmacéutico para la práctica profesional. Conferencia panamericana de educación farmacéutica. 2014;22(1): 8, 15, 16. Disponible en: http://www.observatoriorh.org/pa nama/sites/default/files/webfiles/fulltext/2014/ix_cpef/PlanBasico.pdf

16. Lucchetta R. Capela M. Dos Santos J. Carvalho P. Evaluación del impacto de una intervención educativa en atención farmacéutica y farmacia clínica. Pharmaceutical Care España. 2012; 14(3), 122. Disponible en: http://www.pharmcareesp.com/index. php/PharmaCARE/article/view/76/70

17. Zedillo, P. Reglamento de insumos para la salud. Reforma DOF 04-0298. 2014;III(125): 31.

18. ANAFARMEX. 2013. Disponible en: http://www.anafarmex.com.mx. [Accessed 20 January 2016].

19. Federación Internacional farmacéutica. Buenas prácticas en farmacia: Estándares para la calidad de los servicios farmacéuticos. FIP/ OMS. 2012;1(1):5,6,13,14. Disponible en: https://www.fip.org/www/ uploads/database_file.php?id=334tttable_id=

20. Red Panamericana de armonización de la reglamentación farmacéutica. Grupo de trabajo de farmacovigilancia. Buenas prácticas de farmacovigilancia para las Américas OPS/OMS. Washington, DC. 2010. Pág. 2,5,33. Disponible en: http://apps.who.int/ medicinedocs/documents/s18625es/ s18625es.pdf

21. Ryan R. Santesso N. Lowe D. et al. Las intervenciones para mejorar la seguridad y eficacia en el uso de medicamentos 
humanos: resumen de revisiones sistemáticas. The Cochrane Library, Disponible en: http://www.saludyfarmacos.org/ boletin-farmacos/boletines/ago2014/ p44364

22. Chan, M. Guía para la implementación de estrategias de medicamentos genéricos en los países de américa latina y el caribe como mecanismo para mejorar el acceso a medicamentos. Organización Panamericana de la Salud. 2011; 1(1):63,64,73 y 74. Disponible en: http://apps.who.int/ medicinedocs/documents/s19196es/ s19196es.pdf

23. A Global Competency Framework for Services Provided by Pharmacy Workforce. FIP Pharmacy Education Taskforce. 2010. Disponible en: http:// www.fip.org/files/fip/PharmacyEducation/GbCF\%20booklet.pdf

24. Serie la renovación de la Atención Primaria de Salud OPS/OMS Área de Sistemas y Servicios de Salud HSS-
SP. No. 1. Sistemas de salud basados en APS. Estrategias para el desarrollo de los equipos de APS. Washington, D.C.: OPS, 2008, Pág. 18-23. Disponible en: http://dof.gob.mx/ nota_detalle.php?codigo $=5284236 \mathrm{ft}-$ fecha $=07 / 01$

25. Desgagne, M. Plan Básico de Educación Farmacéutica para el Siglo XXI: Una propuesta de discusión. VII Conferencia Panamericana de Educación Farmacéutica. 2010; 1(1). Disponible en: http://new.paho.org/hq/dmdocuments/2010/plan_basico_de_educacion_farmaceutica_2010.pdf

26. International Pharmaceutical Federation. Declaración de principios de buenas prácticas de educación farmacéutica. Viena. 2000. Pág. 3-5. Disponible: https://www.fip.org/www/ uploads/database_file.php?id=191tttable_id=

27. Organización Panamericana de la Salud/Organización Mundial de la
Salud. Agenda de Salud para las Américas 2008-2017. Washington, D.C.: OPS; 2007. Disponible en: http://www 1.paho.org/hq/dmdocuments/2009/Agenda_Salud_para_ las_Americas_2008-2017.pdf

28. Propuesta regional para la implementación de una estrategia nacional de uso racional de medicamentos. Organización panamericana de la salud. Oficina regional de la OMS. 2010. Pág. 8-48. Disponible en: http://www. paho.org/hq/index.php?option=com docmanttask=doc_download\&tgi$\mathrm{d}=17847$ \& Itemid $=$

29. Baqueiro, F. Programa de acción específico para la protección contra riesgos sanitarios. PAE cofepris. 2013; 1(1):24,46,47. Disponible en: http:// www.cofepris.gob.mx/cofepris/Documents/QueEsCOFEPRIS/PAE\%20 180315.pdf http://www.cofepris.gob. $\mathrm{mx} /$ cofepris/Documents/QueEsCOFEPRIS/prgaccion.pdf 